\title{
Comportement dynamique en torsion et en régime transitoire d'un démarreur de moteur d'avion
}

\author{
Pascal Vernay et Guy Ferraris ${ }^{a}$ \\ Institut national des sciences appliquées de Lyon, Laboratoire de dynamique des machines et des structures, UMR 5006 CNRS, \\ Bâtiment d'Alembert, 8 rue des Sciences, 69621 Villeurbanne Cedex, France
}

Reçu le 11 mai 2004, accepté le 17 octobre 2005

\begin{abstract}
Résumé - Il s'agit de prévoir le comportement dynamique d'un démarreur de moteur d'avion dans le cas d'un redémarrage en vol. Une modélisation du système en torsion est réalisée, prenant en compte les raideurs d'engrenages, les jeux, et la non-linéarité de l'embrayage de type roue libre. Trois types d'éléments sont nécessaires à la modélisation du démarreur : arbre en torsion avec amortisseurs, liaison engrenage avec raideur, jeu et amortisseurs, roue libre embrayage avec raideur non-linéaire. Le système est étudié en régime transitoire dans la phase de réengagement (redémarrage en vol). La montée en vitesse de la turbine à air engendre un couple variable en fonction du temps. Un amortissement localisé sur le moteur d'avion permet de simuler le ralentissement du moteur. Les vitesses des différents éléments, ainsi que les couples supportés par l'arbre de transmission sont examinés. La possibilité de glissement entre les bagues de la roue libre au moment de l'embrayage est simulée, faisant apparaître un surcouple important dans l'arbre de transmission.
\end{abstract}

Mots clés : Torsion / transitoire / dynamique / non-linéaire / démarreur de moteur d'avion

\begin{abstract}
Dynamic behaviour in torsion and in transient motion of an air turbine starter. This study concerns the dynamic behaviour of an air turbine starter during re-engagement in flight. Torsional behaviour is modelled, taking into account gears stiffness gaps and the non-linear characteristics of the sprag-type clutch. Three type of elements are needed: shaft in torsion with dampers, gaps and dampers, free wheel with non-linear stiffness. The system is studied during the re-engagement transient phase. Increasing turbine speed of rotation induces a time varying torque. A damper situated on the plane engine allows slow-down simulations. Speeds of the different elements as well as torques applied on the transmission shaft are examined. Sliding possibility between races of the free wheel during engagement are simulated, showing a significant over-torque within the transmission shaft.
\end{abstract}

Key words: Torsion / transient motion / dynamics / non-linear / air turbine starter

\section{Introduction}

Les démarreurs à air des réacteurs des avions de combat sont parfois amenés à redémarrer les réacteurs en plein vol. En effet, au cours de manœuvres extrêmes un réacteur peut caler et le démarreur doit alors le relancer, cette action s'appelle le réengagement. Lors de certains réengagements dont les conditions ne sont pas clairement identifiées, des ruptures de l'arbre lié au réacteur ont été constatées. Pour maîtriser ces risques, la connaissance du comportement dynamique en torsion du système s'avère indispensable.

Des modèles simples de prévision du comportement dynamique de démarreurs ont été développés par $[1,2]$. Ces systèmes, à deux degrés de liberté, représentent

\footnotetext{
a Auteur correspondant : guy.ferraris@insa-lyon.fr
}

les inerties de la turbine et du réacteur liées par une raideur linéaire équivalente. Ces modèles ne prennent pas en compte les inerties intermédiaires et les étages de réduction de l'arbre de transmission, de plus le réengagement est simplement caractérisé par des conditions initiales en vitesses non nulles. Dans [3], une étude théorique sur le comportement d'ensemble et une théorique et expérimentale sur le comportement de l'embrayage par roue libre ont été réalisées.

L'objectif de cette étude est de déterminer le comportement dynamique en torsion d'un démarreur de réacteur d'avion au cours d'un réengagement afin de définir les causes probables de rupture. Pour cela, une modélisation du mécanisme est réalisée, le comportement en régime transitoire est alors déterminé de même que les efforts supportés par chacun des éléments. 


\section{Modélisation}

Il s'agit de modéliser le démarreur en tenant compte de sa liaison avec le réacteur.

Le démarreur (Fig. 1) est composé d'une turbine, d'un réducteur pouvant comporter plusieurs trains épicycloïdaux et d'un embrayage type roue libre permettant la liaison avec le réacteur. Lors de ces réengagements, l'accouplement brutal crée, dans l'arbre de transmission, des surcouples qui peuvent entraîner des dommages irrémédiables.

Pour la modélisation, trois modèles élémentaires sont utilisés : un modèle d'arbre en torsion, un modèle d'engrenage avec ou sans jeu de fonctionnement et un modèle de roue libre.

\section{1 Élément discret d'arbre}

Cet élément à deux nœuds (Fig. 2) est constitué d'une raideur constante, de deux inerties ponctuelles et de deux amortisseurs pouvant être non-linéaires.

Le système (Fig. 2) montre un amortisseur constant au nœud 1 et un amortisseur non-linéaire au nœud 2, cependant tout autre combinaison est possible. Les équations du mouvement correspondantes sont :

$$
\left\{\begin{array}{l}
I_{1} \ddot{\theta}_{1}+C l \times \dot{\theta}_{1}+K\left(\theta_{1}-\theta_{2}\right)=C e_{1} \\
I_{2} \ddot{\theta}_{2}+C n l \times \dot{\theta}_{1}^{n}+K\left(\theta_{2}-\theta_{1}\right)=C e_{2}
\end{array}\right.
$$

Le terme relatif à l'amortissement non-linéaire de la deuxième équation peut se mettre sous la forme : $C n l \times$ $\left|\dot{\theta}_{1}\right|^{n-1} \times \dot{\theta}_{1}$, afin que le couple résistant correspondant reste opposé à la vitesse.

Les inerties $I_{1}$ et $I_{2}\left(\mathrm{~kg} . \mathrm{m}^{2}\right)$, peuvent également inclure l'inertie d'un disque en bout d'arbre. Par exemple $I_{1}$ peut représenter la demi-inertie de l'arbre plus l'inertie de la turbine alors que $I_{2}$ ne représente que la demi-inertie de l'arbre. $K\left(\mathrm{Nm} \cdot \mathrm{rad}^{-1}\right)$ est la raideur de l'arbre, elle est en principe égale à $\frac{G J}{L} . C l\left(\mathrm{Nms}_{\mathrm{rad}}{ }^{-1}\right)$ représente l'amortissement linéaire visqueux, et l'ensemble $C n l \times\left|\dot{\theta}_{1}\right|^{n-1}$ $\left(\mathrm{Nms}_{\mathrm{rad}}{ }^{-1}\right)$ l'amortissement non-linéaire. $C e_{1}$ et $C e_{2}$ sont les couples extérieurs fonction du temps appliqués aux nouds 1 et 2 . Ces couples sont de la forme indiquée figure 3 , la partie $A B$ peut être linéaire ou parabolique. Ces couples peuvent aussi être constants (partie $B C$ uniquement).

\section{2 Élément d'engrenage}

L'étage de réduction est modélisé en tenant compte des déformations de denture des engrenages et des jeux de fonctionnement. Des jeux apparaissent aussi dans la liaison par cannelures entre la sortie du démarreur et l'entrée du réacteur. Ces modèles sont développés dans de nombreux articles. [4] fait apparaître une raideur de denture périodique et tient compte des défauts de profil des dents. Un modèle simple est directement issu de $[5,6]$,

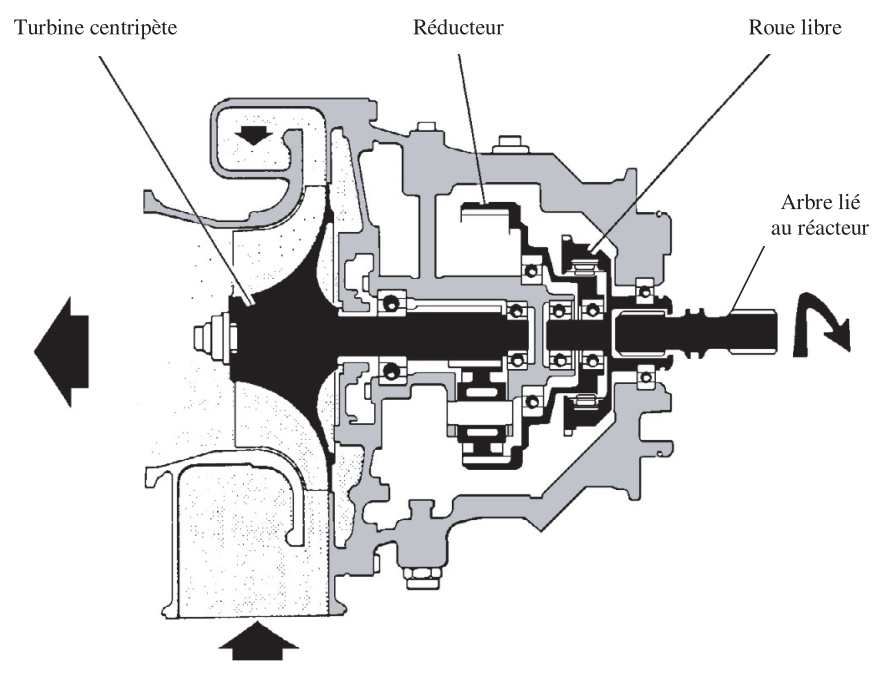

Fig. 1. Schéma de fonctionnement d'un démarreur.

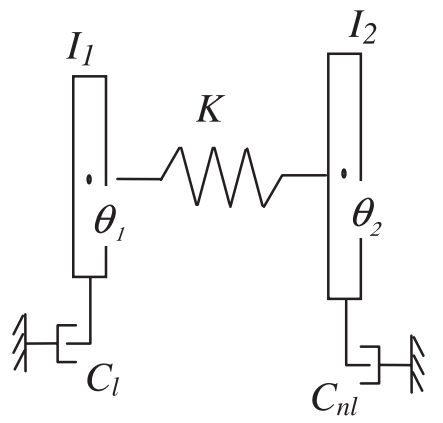

Fig. 2. Modèle d'arbre en torsion à 2 ddl.

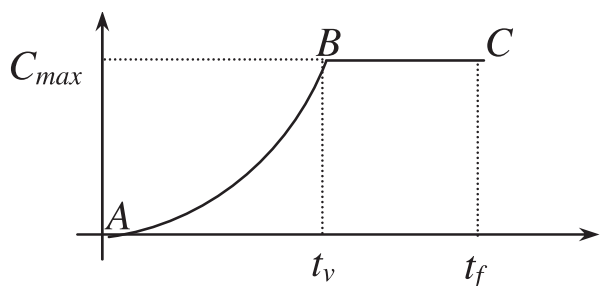

Fig. 3. Représentation des couples extérieurs.

il permet de définir un amortissement de contact à partir de la théorie des chocs [7].

L'élément d'engrenage (Fig. 4) est modélisé par les inerties $I_{1}$ et $I_{2}$ liées par la raideur des dentures $K_{\mathrm{d}}$ et l'amortissement de contact $A m_{2}$ lorsque le jeu est consommé. Un amortissement $A m_{1}$ a été ajouté pour tenir compte d'une dissipation d'énergie due au barbotage des engrenages dans un bain d'huile. Cet amortissement $A m_{1}$ est beaucoup plus faible que l'amortissement $\mathrm{Am}_{2}$. Avec

$K_{\mathrm{d}}$ : raideur de la dent en prise en N.m ${ }^{-1}$.

$R_{1}$ : rayon de la roue menante en mètre.

$R_{2}$ : rayon de la roue menée en mètre.

$j$ : jeu de fonctionnement en mètre.

$A m_{1}$ : amortissement visqueux relié à la vitesse relative d'un nœud par rapport à l'autre $\left(\mathrm{Ns}_{\mathrm{m}}{ }^{-1}\right)$.

$A m_{2}$ : amortissement visqueux de contact. 


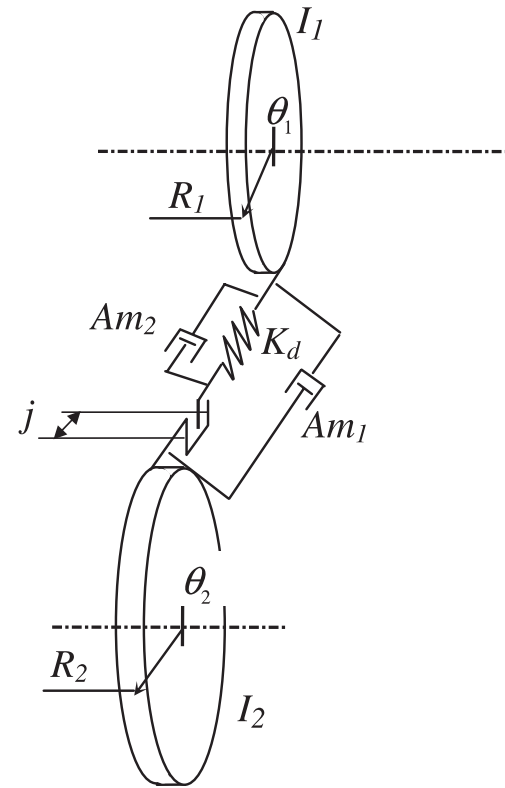

Fig. 4. Modèle d'engrenage à 2 ddl.

Trois états du système sont identifiés selon la position relative des dentures, figure 5

$$
\begin{gathered}
R_{1} \theta_{1}+R_{2} \theta_{2} \geq j \quad 0<R_{1} \theta_{1}+R_{2} \theta_{2}<j \\
R_{1} \theta_{1}+R_{2} \theta_{2} \leq 0
\end{gathered}
$$

Les équations du mouvement à l'état 1 du modèle décrit figure 4 sont :

$$
\left\{\begin{array}{c}
I_{1} \ddot{\theta}_{1}+A m_{1} R_{1}\left(R_{1} \dot{\theta}_{1}+R_{2} \dot{\theta}_{2}\right)+A m_{2} R_{1}\left(R_{1} \dot{\theta}_{1}+R_{2} \dot{\theta}_{2}\right) \\
\quad+K_{\mathrm{d}} R_{1}\left(R_{1} \theta_{1}+R_{2} \theta_{2}-j\right)=C e_{1} \\
I_{2} \ddot{\theta}_{2}+A m_{1} R_{2}\left(R_{1} \dot{\theta}_{1}+R_{2} \dot{\theta}_{2}\right)+A m_{2} R_{2}\left(R_{1} \dot{\theta}_{1}+R_{2} \dot{\theta}_{2}\right) \\
\quad+K_{\mathrm{d}} R_{2}\left(R_{1} \theta_{1}+R_{2} \theta_{2}-j\right)=C e_{2}
\end{array}\right.
$$

$C e_{1}$ et $C e_{2}$ sont de la même forme que ceux décrits précédemment.

Lorsque la dent se trouve à l'intérieur du jeu de fonctionnement, état 2, les équations du mouvement sont celles du système (4) dans lequel $A m_{2}=0$ et $K_{\mathrm{d}}=0$.

Les équations du mouvement à l'état 3 sont :

$$
\left\{\begin{array}{l}
I_{1} \ddot{\theta}_{1}+A m_{1} R_{1}\left(R_{1} \dot{\theta}_{1}+R_{2} \dot{\theta}_{2}\right)+A m_{2} R_{1}\left(R_{1} \dot{\theta}_{1}+R_{2} \dot{\theta}_{2}\right) \\
\quad+K_{\mathrm{d}} R_{1}\left(R_{1} \theta_{1}+R_{2} \theta_{2}\right)=C e_{1} \\
\ddot{I}_{2} \ddot{\theta}_{2}+A m_{1} R_{2}\left(R_{1} \dot{\theta}_{1}+R_{2} \dot{\theta}_{2}\right)+A m_{2} R_{2}\left(R_{1} \dot{\theta}_{1}+R_{2} \dot{\theta}_{2}\right) \\
\quad+K_{\mathrm{d}} R_{2}\left(R_{1} \theta_{1}+R_{2} \theta_{2}\right)=C e_{2}
\end{array}\right.
$$

Ce modèle permet également de modéliser un engrenage à denture intérieure ainsi que des cannelures en considérant un rayon négatif.

Pour modéliser un train épicyclö̈dal dont le porte satellite est lié au bâti, deux étages de réduction doivent être mis l'un à la suite de l'autre.

\subsection{Modèle de roue libre}

Le modèle de roue libre est présenté figure $6, I_{1}$ représente l'inertie de la bague intérieure de la roue libre et des pièces qui lui sont solidaires. De même $I_{2}$ est l'inertie de la bague extérieure et des pièces de liaison. $K_{r l}$ est la raideur non-linéaire de l'embrayage.

Les équations du mouvement correspondant au modèle figure 6 sont :

$$
\left\{\begin{array}{l}
I_{1} \ddot{\theta}_{1}+K r l \times\left(\theta_{1}-\theta_{2}\right)^{n e}=C e_{1} \\
I_{2} \ddot{\theta}_{2}+K r l \times\left(\theta_{2}-\theta_{1}\right)^{n e}=C e_{2}
\end{array}\right.
$$

Afin de respecter le signe du couple élastique correspondant, le terme relatif à la raideur non-linéaire peut se mettre sous la forme : $K r l \times\left|\theta_{1}-\theta_{2}\right|^{n e-1} \times\left(\theta_{1}-\theta_{2}\right)$.

En configuration embrayé $\left(\left(\theta_{1}-\theta_{2}\right) \geq 0\right)$, la raideur, $K r l \times\left|\theta_{1}-\theta_{2}\right|^{n e-1}\left(\mathrm{Nm} \cdot \mathrm{rad}^{-1}\right)$, varie en fonction des déplacements angulaires. En configuration libre $\left(\dot{\theta}_{1}<\dot{\theta}_{2}\right)$ $K r l$ est mis à zéro, les deux inerties sont découplées.

Le passage du mode libre au mode embrayé est réalisé quand la vitesse du nœud 1 rejoint celle du nœud 2. À cet instant l'angle de rotation du nœud 2 est égalé à celui du nœud 1, les écarts angulaires entre les nœuds des éléments se situant après la bague extérieure de la roue libre sont conservés.

Le passage du mode embrayé au mode libre est réalisé quand l'angle de rotation relatif des bagues de la roue libre devient nul : $\left(\theta_{2}-\theta_{3}\right)=0$.

Tous les éléments décrits sont assemblés et les équations générales du mouvement sont de la forme :

$$
I \ddot{\theta}+C(\dot{\theta}) \dot{\theta}+K(\dot{\theta}, \theta) \theta=C e(t)
$$

La méthode numérique de Runge Kutta du quatrième ordre est utilisée pour la résolution des équations du mouvement. La difficulté de mise au point de la méthode pas à pas est due à la gestion des multiples non-linéarités dues aux jeux de fonctionnement. Plusieurs changements d'état peuvent avoir lieu en même temps.

Dès qu'un changement d'état est détecté entre $t$ et $t+\Delta t$, le pas de temps $\Delta t$ est divisé par 10 et la simulation recommence à partir de $t$ sans tenir compte du calcul à $t+\Delta t$. Un changement d'état est déterminé avec une précision de $\Delta t / 1000$. Dans cet intervalle de temps $(\Delta t / 1000)$ plusieurs changements d'état peuvent se produire « simultanément ».

\section{Simulation}

Le logiciel développé permet de simuler le comportement dynamique du système, les éléments décrits dans la modélisation sont assemblés dans l'ordre voulu par l'utilisateur.

La modélisation du démarreur est présentée figure 7 . L'essentiel des valeurs numériques est présenté tableau 1.

- Le couple moteur au nœud 1 atteint son maximum en $4 \mathrm{~s}$. 
Etat 1

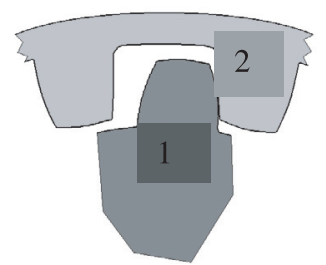

Etat 2

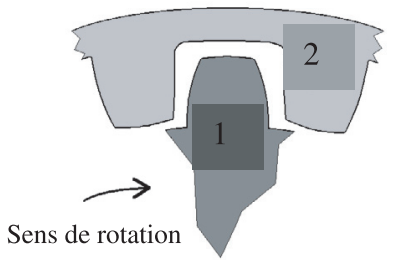

Etat 3

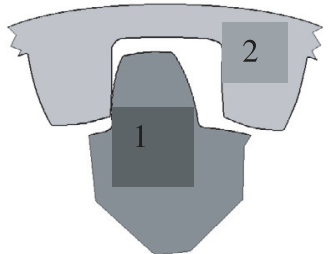

Fig. 5. Schéma de position des dentures.

Tableau 1. Données numériques du modèle à $10 \mathrm{ddl}$ pour un réengagement.

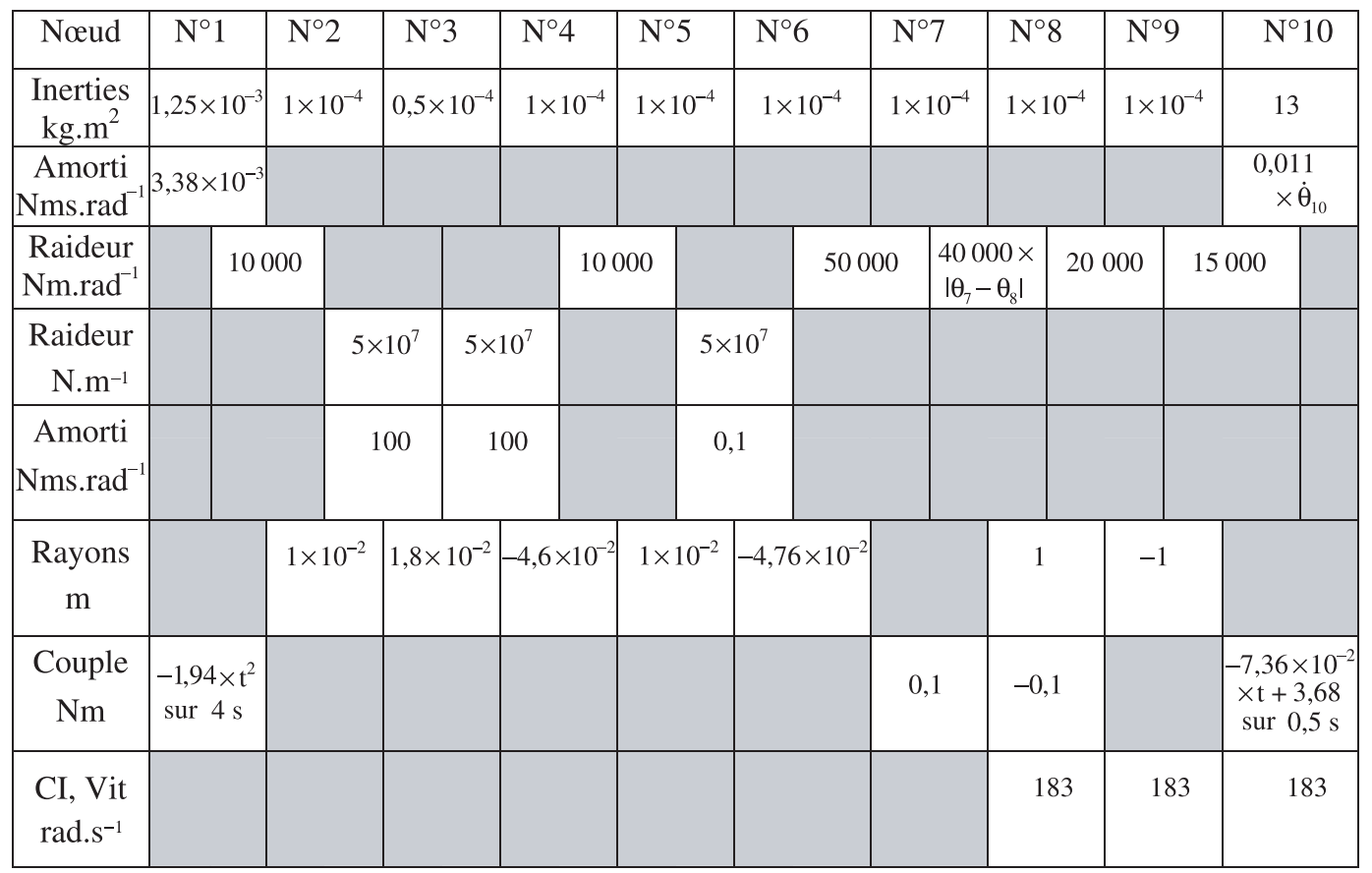

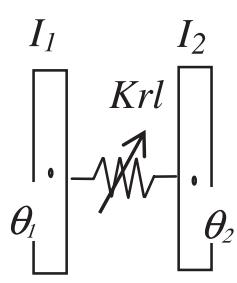

Fig. 6. Modèle de roue libre à 2 ddl.

- Dans le cas d'un réengagement, le couple moteur au nœud 10 équilibre à l'instant initial le couple résistant provenant de l'amortissement visqueux au même noud et s'annule en $0,5 \mathrm{~s}$. De plus la vitesse initiale du réacteur (nœuds 8, 9 et 10) est égale à

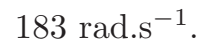

- Les couples aux nouds 7 et 8 proviennent du frottement sec de l'embrayage en mode libre. Sa valeur est environ $1 / 5000^{\text {ième }}$ du couple nominal.

- La valeur des jeux des engrenages est de $5 \times 10^{-4} \mathrm{~m}$, et celui des cannelures est de 0,1 rad.

- L'amortissement permanent $A m_{12}$ au niveau des cannelures est de $10^{-3} \mathrm{Nms}_{\text {.rad }}^{-1}$.

\subsection{Simulation d'un réengagement}

La figure 8 représente l'évolution des vitesses du système au cours du temps. Les trois rapports de réduction apparaissent nettement.

La figure 9 montre les chocs et les mouvements des engrenages. Au début de la simulation, avant que le système embraye, les jeux sont complètement rattrapés. Par contre, à l'instant de l'accrochage, le jeu des cannelures (0,1 rad) est entièrement traversé, figure 10 , créant ainsi un léger retard à l'embrayage, ce qui a pour conséquence d'augmenter les amplitudes des premières oscillations du couple transmis, figure 11, et de provoquer un léger désembrayage.

Ces vibrations ne sont pas suffisamment importantes pour expliquer les phénomènes de rupture.

\subsection{Simulation d'un embrayage retardé}

[8] et [9] ont fait apparaître des problèmes de glissement au niveau des bagues de la roue libre à l'instant de l'embrayage. Dans ce cas, soit le système réaccroche, 


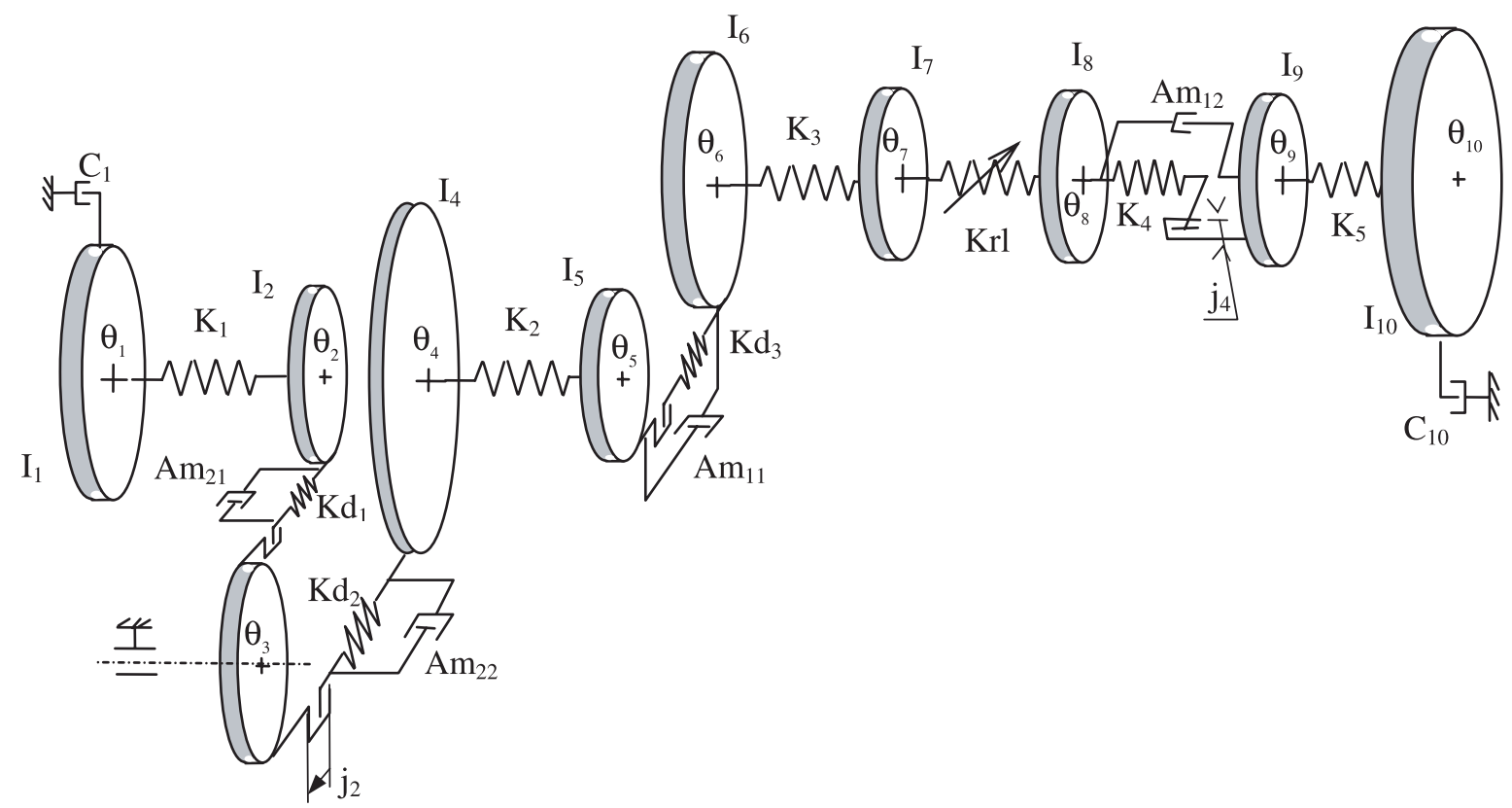

Fig. 7. Modèle à 10 degrés de liberté.

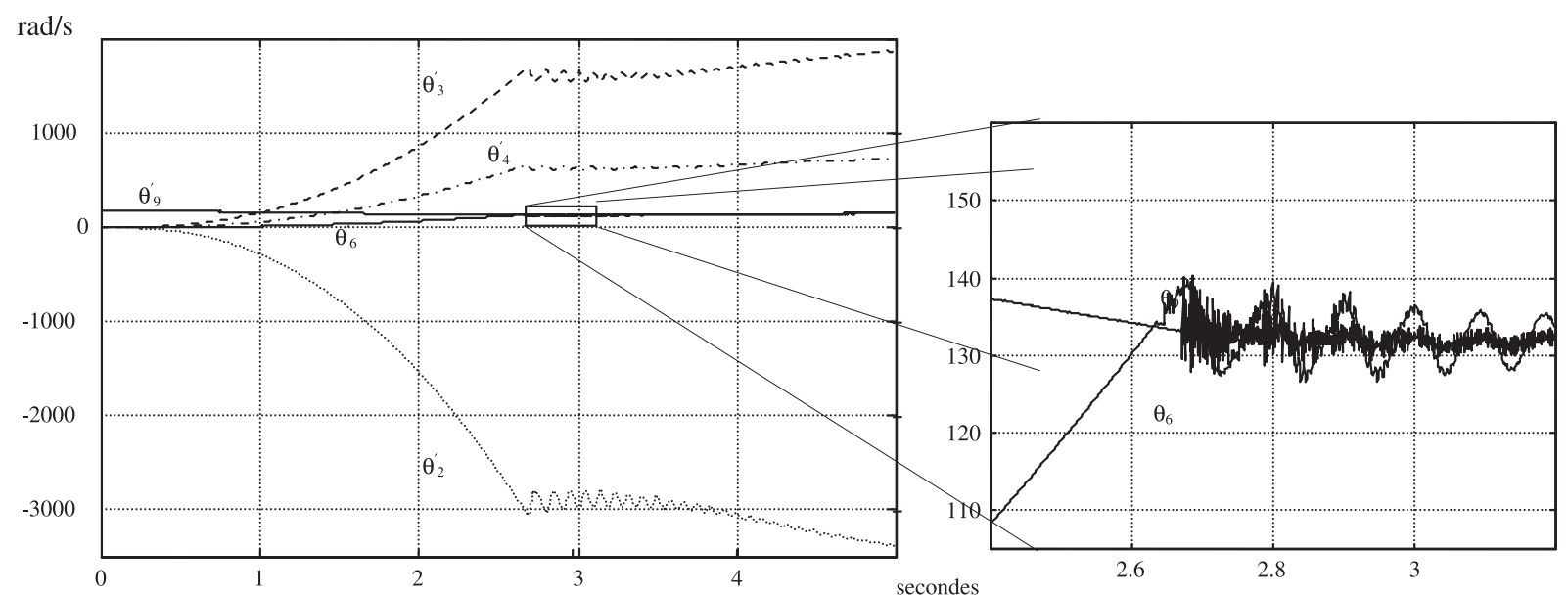

Fig. 8. Vitesses de rotation.

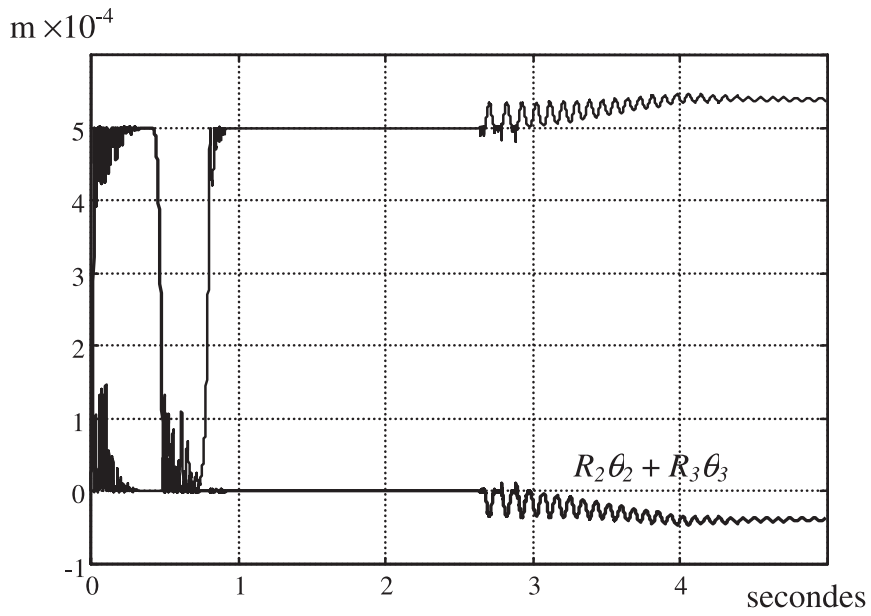

Fig. 9. Position des dents des deux premiers engrenages.

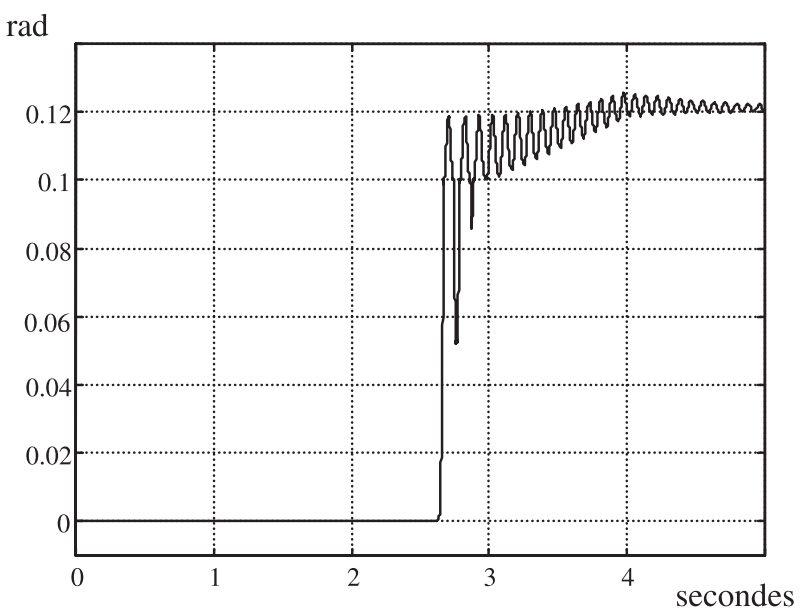

Fig. 10. Position relative des cannelures. 


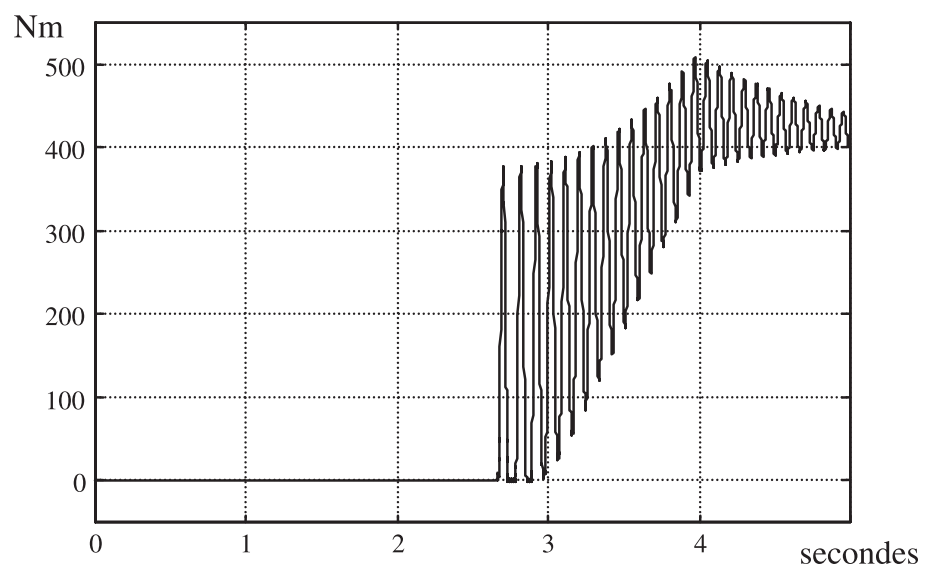

Fig. 11. Couple transmis au réacteur.

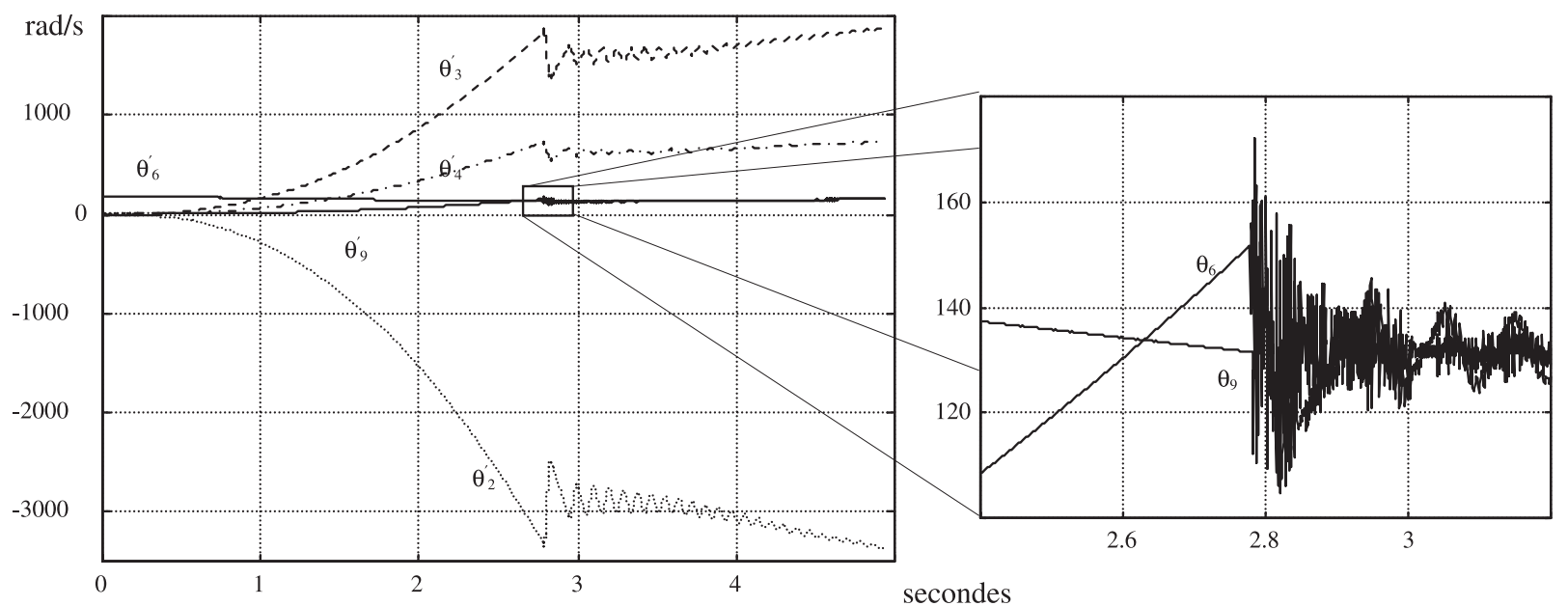

Fig. 12. Vitesses de rotation, embrayage retardé.

soit le redémarrage du réacteur est impossible. Le premier phénomène peut être simplement modélisé par un retard d'embrayage, un décalage en vitesse $\Delta V$ est alors imposé. La roue libre accroche quand la vitesse de la bague intérieure est supérieure de $\Delta V$ à celle de la bague extérieure. [10] met en évidence, expérimentalement, que des phénomènes de glissement peuvent apparaître, en mode embrayé, lorsque la roue libre est dans un environnement vibratoire.

La simulation présentée est réalisée pour $\Delta V=$ $20 \mathrm{rad} . \mathrm{s}^{-1}$. Ce décalage en vitesse correspond environ à un tour de glissement.

$\Delta V$ apparaît nettement sur l'agrandissement de la figure 12. Le temps de glissement est supérieur à $0,1 \mathrm{~s}$.

Cet embrayage violent entraîne de nombreuses oscillations à l'intérieur des jeux de fonctionnement, figures 13 et 14. De plus le système décroche nettement avant de rester définitivement embrayé.

Le retard de l'embrayage ajouté à celui dû à la position des cannelures, provoque un surcouple important au moment du réaccrochage, figure 15. La valeur maximale (1200 Nm) est le triple du couple nominal.

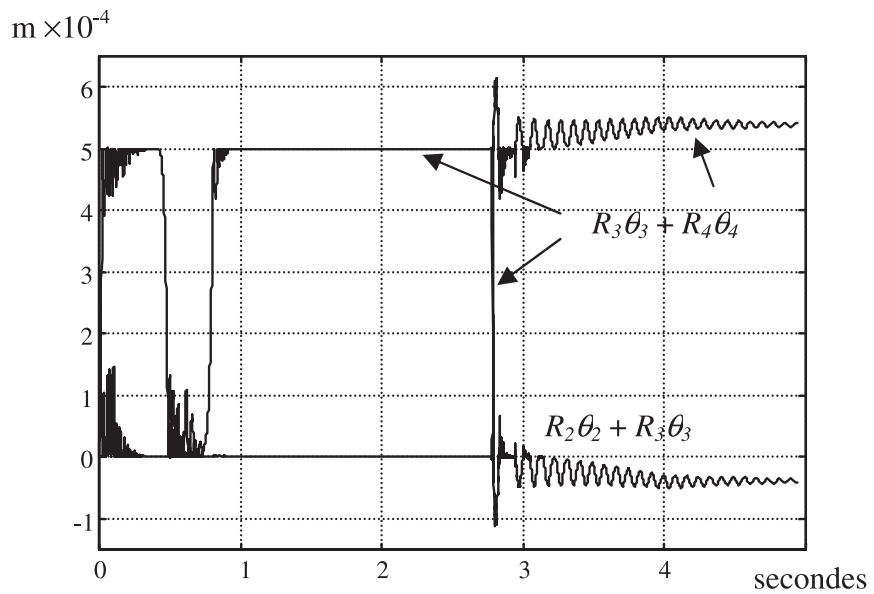

Fig. 13. Position des deux premiers engrenages, embrayage retardé.

\section{Conclusion}

Avec le logiciel, le comportement dynamique d'un démarreur lié à un réacteur peut-être entièrement simulé à partir d'un assemblage de modèles linéaires et 


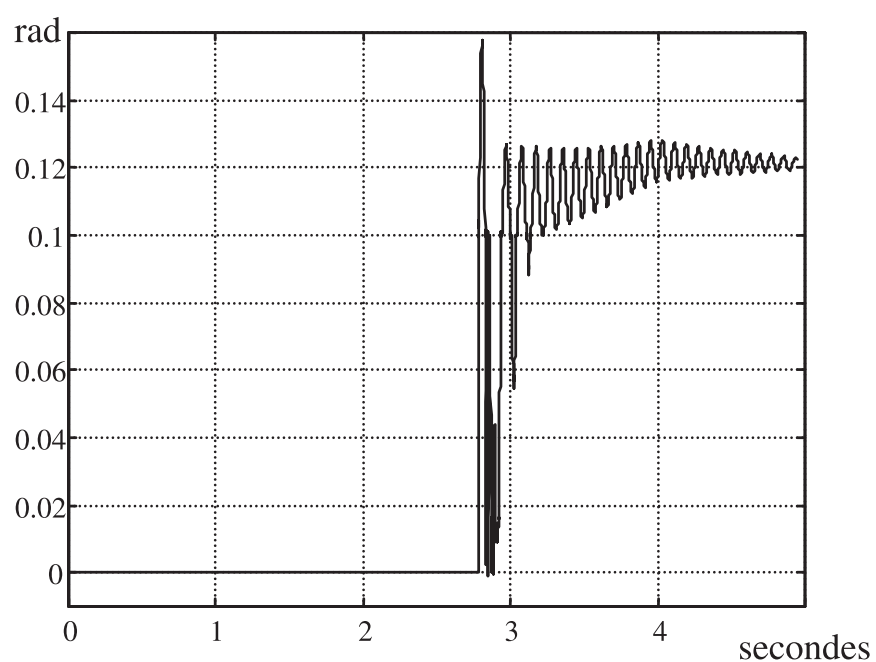

Fig. 14. Position relative des cannelures, embrayage retardé.

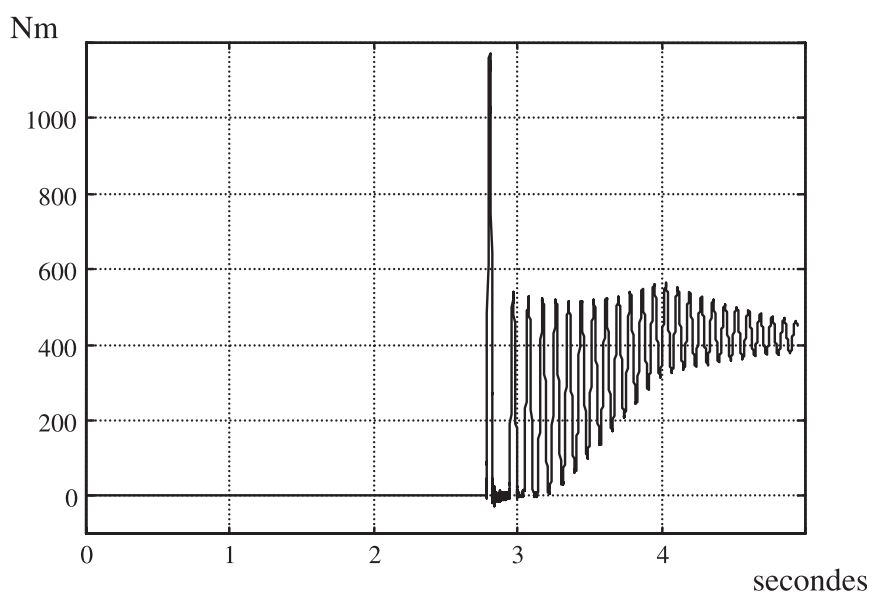

Fig. 15. Couple transmis au du réacteur, embrayage retardé.

non-linéaires. Les modèles élémentaires existants sont : un modèle d'arbre en torsion, un modèle d'engrenage avec ou sans jeu de fonctionnement et un modèle de roue libre. Une application sur un système concret est présentée. Un surcouple important a été observé dans le cas où un embrayage retardé a été imposé. Ce retard est dû à une période de glissement de la roue libre. Cette période de glissement doit être déterminée expérimentalement pour chaque type d'embrayage considéré. Une expérimentation [10] a permis d'observer ces phases de glissement et de valider le modèle développé.

\section{Références}

[1] Guide for determining engine starter drive torque requirements, New York: Society of Automative Engineers, 1962, Aerospace Information Report $\mathrm{N}^{\circ}$ AIR 781

[2] N. Eiroy, Peak transient torques in pneumatic starterengine systems, Garett Pneumatic Systems, rapport $\mathrm{N}^{\circ} 841510$

[3] P. Vernay, Comportement dynamique en torsion et en régime transitoire d'un démarreur de moteur d'avion, Thèse de doctorat INSA-Lyon, 1999

[4] P. Velex, D. Berthe, Eccentricity and meshing contributions to the dynamic tooth loading and gearing trains, 3rd IFToMM Rotordynamics Conf., Lyon, 1990, pp. 219-224

[5] P. Couderc, Comportement dynamique des chaînes de transmission automobiles, Thèse de doctorat INSA-Lyon, 1997

[6] A.S. Yigit, R.A. Scott, A.G. Ulsoy, Dynamics of a radially rotating beam whith impact : part 1 : théorical and computational model, 12th Biennal conference on mechanical vibration and noise, ASME, Montreal, Quebec, Canada, Sep. 17-21, 1989, 18-3, pp. 77-82

[7] J.P. Brossard, D. Cesari, Les théories du choc, tomes 1 et 2, Lyon : INSA, 1978

[8] H.J. Peeken, P.W. Gold, Couplings and Clutches State of the art, Proc. Intl. Conf. on Gears, Dusseldorf Germany, Apr. 22-24, 1996, pp. 47-60

[9] F.C. Williams, D. Tipping, T.A. Henry, An improved sprag clutch, World Congr. on the Theory of Mach. and Mech., 4th, Univ. of Newcastle upon Tyne, Engl., Sep. 8-12 1975, pp. 593-598

[10] P. Vernay, G. Ferraris, A. Delbez, P. Ouplomb, Transient behavoiur of a sprag-type over-running clutch: an experiment study, J. Sound Vib. 248 (2001) 567-572 